\title{
All-Optical Image Recognition Using Metamaterials
}

\author{
$\underline{\text { M. Papaioannou }}^{1}$, E. Plum ${ }^{1}$, Edward T. F. Rogers ${ }^{1,2}$, João Valente ${ }^{1}$, and Nikolay I. Zheludev ${ }^{1,3}$ \\ ${ }^{1}$ Optoelectronics Research Centre and Centre for Photonic Metamaterials, \\ University of Southampton, SO17 1BJ, UK \\ ${ }^{2}$ Institute for Life Sciences, University of Southampton, Southampton SO17 1BJ, UK \\ ${ }^{3}$ Centre for Disruptive Photonic Technologies and The Photonics Institute, \\ Nanyang Technological University, Singapore 637371, Singapore \\ mcp1g13@soton.ac.uk,tel: +442380592956
}

\begin{abstract}
Coherent interaction of waves on a thin absorbing film allows performance of ultrafast, low energy logical functions with two-dimensional images, adaptive filtering and pattern recognition that we demonstrate with a plasmonic metamaterial absorber.

OCIS codes: (100.3005) Image recognition devices; (160.3918) Metamaterials; (200.3760) Logic-based optical processing
\end{abstract}

\section{Introduction}

We perform all-optical image recognition based on the effective nonlinearity of a system where a lossy thin film or metamaterial of subwavelength thickness is translated relative to a standing wave. Perfect transmission at electric field nodes and perfect absorption at anti-nodes result from suppression and enhancement of the light-matter interaction, respectively [1]. This all-optical effect enables logical operations between optical signals, promising alloptical data processing at arbitrarily low power and with 10s of THz bandwidth [2-4].

Here we present the first experimental metasystem for all-optical image recognition that is underpinned by the coherent interaction of light with light on a metamaterial. A target image and test images are projected on opposite sides of an absorbing metamaterial (Fig. 1). Coherent light-matter interaction will take place across features that are common in both images. At an electric field node, image similarities will be revealed due to perfect transmission of matching features (Fig. 1a). On the other hand, at the antinode, only image differences will be detected due to perfect absorption of common image features (Fig. 1b). This all-optical approach allows identification of matching images and quantifies the degree of image mismatch.
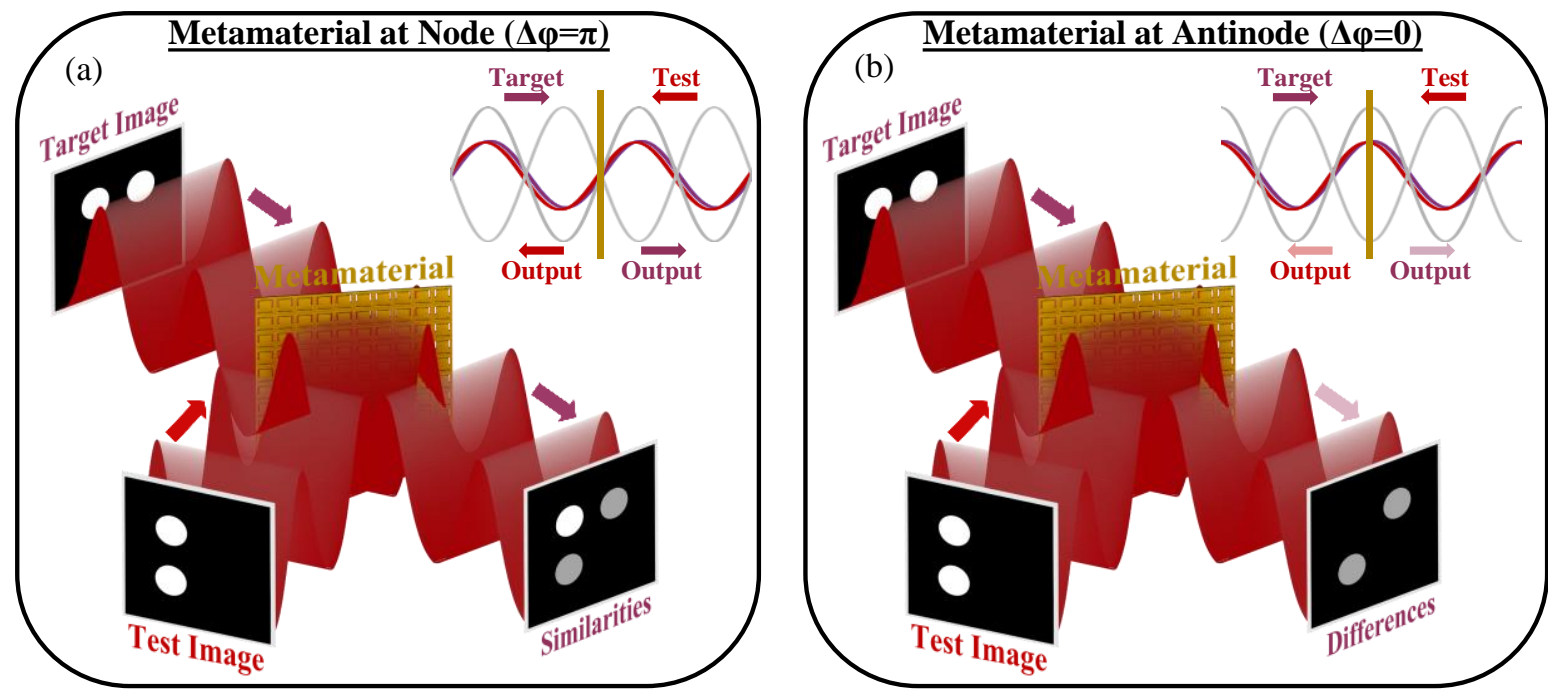

Fig. 1. All-optical image recognition metasystem: Test and target images are projected onto an absorbing metamaterial. Elimination or enhancement of absorption occurs across common image features where the coherent illuminating beams interact. Matching image features are (a) perfectly transmitted at nodes (bright spot) and (b) perfectly absorbed at antinodes revealing the image (a) similarities and (b) differences.

\section{Results}

All-optical coherent image recognition is performed on an absorptive free-standing gold metamaterial of subwavelength thickness perforated with a periodic array of split ring resonators. The metamaterial absorbs close to $50 \%$ of a single illuminating beam. Target and test images are projected on either side of the metamaterial. The output light is monitored by a CCD camera which images the metamaterial plane. 
We compare binary patterns of bright features occupying 8 positions in a $4 \times 4$ grid ( $1^{\text {st }}$ row in Fig. 2 ).

Identification of the target image pattern (indicated by green circles in Fig. 2) amongst a set of test images is the experimental goal. Here we show results for test images corresponding to $0 \%, 50 \%$ and $100 \%$ image agreement. Any type of optical data streams could be represented in this form. Destructive interference prevents absorption of matching image features if the target and test images reach the metamaterial with $\pi$ phase difference (metamaterial at a node) and therefore matching dots appear bright ( $2^{\text {nd }}$ row in Fig. 2$)$. In contrast, only differences are detected if the images are projected in phase (metamaterial at an antinode) due to complete absorption of overlapping features, ( $3^{\text {rd }}$ row in Fig. 2$)$.

Alternatively, the overall detected power modulation between nodes and antinodes can be used to identify the target image and to quantify the image agreement. Node to antinode translation modulates the total detected optical power when there is a non-zero image agreement. Moreover, the phase-dependent power fluctuation is directly proportional to image agreement. Therefore, image recognition can also be accomplished with a photodetector monitoring the total output power level or/and the level of power fluctuation between nodes and antinodes.

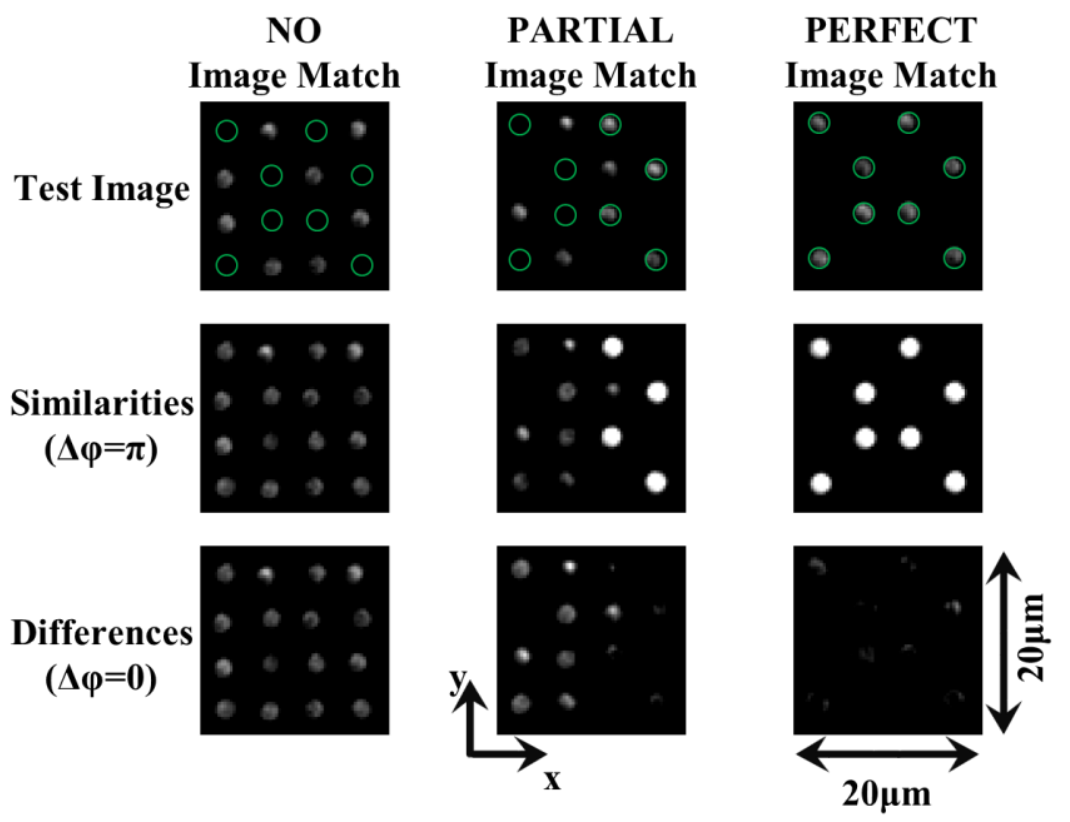

Fig. 2. Experimental image recognition. Test images ( $1^{\text {st }}$ row) are compared to the target image (indicated by green circles) on an absorbing metamaterial using coherent light of $785 \mathrm{~nm}$ wavelength. Overlapping image features are perfectly transmitted ( $2^{\text {nd }}$ row) or fully absorbed $\left(3^{\text {rd }}\right.$ row) depending on the position of the metamaterial with respect to the standing wave. All images are presented on the same grayscale.

\section{Conclusions}

The first experimental metasystem for all-optical image recognition is demonstrated employing coherent lightmatter interactions on an absorptive metamaterial. Image similarities and differences are quantitatively detected with diffraction-limited resolution. Our approach can also be applied to moving images as well as massively parallel data streams and is in principle compatible with $10 \mathrm{~s} \mathrm{of} \mathrm{THz}$ frame rates or data rates as well as quantum-level intensities.

\section{References}

[1] J. Zhang, K. F. MacDonald, and N. I. Zheludev, "Controlling light-with-light without nonlinearity”, Light: Science and Applications 1, e18, (2012).

[2] M. Papaioannou, E. Plum, J. Valente, E. Rogers, and N. I. Zheludev, "Two-dimensional control of light with light on metasurfaces", Light: Science and Applications 5, e16070, (2016).

[3] X. Fang, M. L. Tseng, J. Y. Ou, K. F. MacDonald, D. P. Tsai, and N. I. Zheludev, "Ultrafast all-optical switching via coherent modulation of metamaterial absorption", Appl. Phys. Lett. 104, p. 141102, 2014.

[4] T. Roger, S. Vezzoli, E. Bolduc, J. P. Valente, J. J. F. Heitz, J. Jeffers, C. Soci, J. Leach, C. Couteau, N. I. Zheludev, and D. Faccio, "Coherent perfect absorption in deeply subwavelength films in the single-photon regime", Nat. Commun. 6, 7031, 2015. 